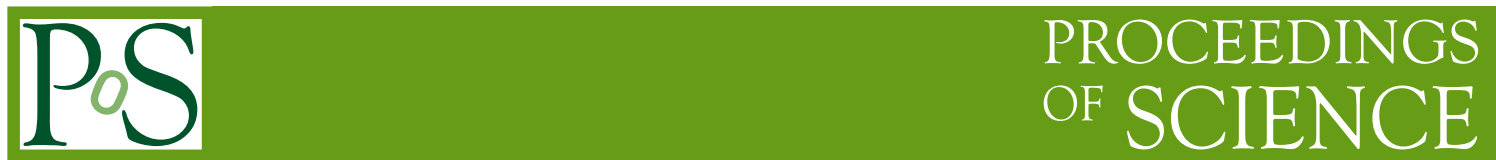

\title{
Searches in s-channel single top quark production at ATLAS
}

\section{Barbara Alvarez Gonzalez*}

On behalf of the ATLAS Collaboration

Michigan State University

E-mail: balvarez@fnal.gov

Two searches with the ATLAS detector at the Large Hadron Collider in proton-proton collisions at $\sqrt{s}=7 \mathrm{TeV}$ are presented: one for Standard Model single top-quark production in the s-channel, and the other for tb resonances. The analyses use the same event selection and background estimation techniques. An observed (expected) upper limit at $95 \%$ confidence level on the SM s-channel single top-quark production cross-section of $\sigma<26.5(20.5) \mathrm{pb}$ is obtained using a cutbased analysis. The result of the $t b$ resonances search in the same final state is also given. This result is translated into a lower bound on a right-handed $W_{R}^{\prime}$ boson mass, giving $m_{W_{R}^{\prime}}>1.13 \mathrm{TeV}$ at 95\% credibility-level.

36th International Conference on High Energy Physics

4-11 July 2012

Melbourne, Australia

\footnotetext{
*Speaker.
} 


\section{Introduction}

Studies of the $t b$ final state are particularly interesting at the Large Hadron Collider (LHC): the Standard Model (SM) s-channel single top-quark production mode itself has not been observed yet and the search for this process is sensitive to several models of new physics [1].

SM single top-quark production proceeds through three different mechanisms: t-channel exchange of a W boson [ $₫$ ] $\left(\sigma=64.57_{-2.62}^{+3.32} \mathrm{pb}\right.$ at $\left.7 \mathrm{TeV}\right)$, associated production of a top-quark and a $\mathrm{W}$ boson [5] $\left(\sigma=15.74_{-1.36}^{+1.34}\right.$ at $\left.7 \mathrm{TeV}\right)$, and s-channel production and decay of a virtual $W$ boson [6] $\left(\sigma=4.63_{-0.27}^{+0.29}\right.$ at $\left.7 \mathrm{TeV}\right)$ as shown in Fig. 1 .

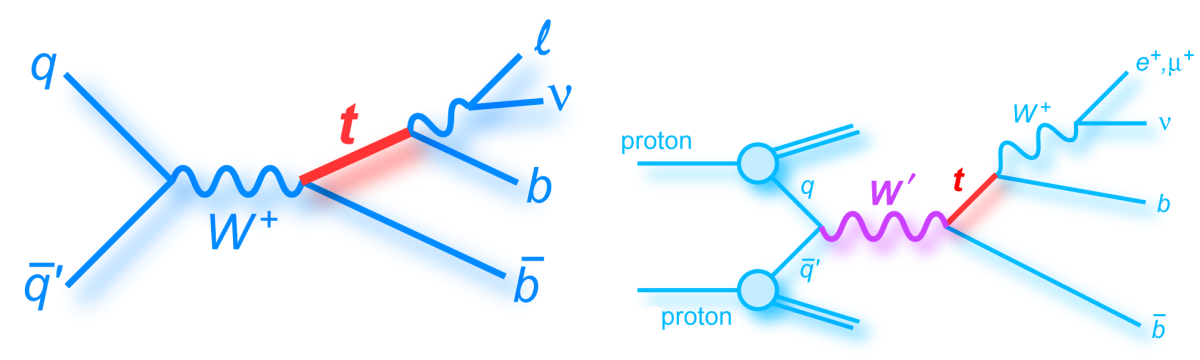

Figure 1: Leading order diagrams corresponding to SM s-channel single top (left) and $W^{\prime} \rightarrow t b$ (right) production.

\section{Detector Description}

The ATLAS detector [2] is a multi-purpose particle physics detector at the LHC at CERN with a forward-backward symmetric cylindrical geometry and nearly $4 \pi$ coverage in solid angle. The innermost part of the ATLAS detector, a tracking system in a $2 \mathrm{~T}$ axial magnetic field, measures the momentum of the charged particles produced in the collisions. Outside of the solenoid are the calorimeter subsystems, which measure the electron, photon, and hadronic jet energies, and the muon spectrometer which is used to identify and measure the momentum of muons in a toroidal magnetic field. A three-level trigger system [3] reduces the event rate and selects the events for analysis. For the analyses presented here, candidate events are identified using single high-transverse momentum electron and muon triggers and stringent detector and data quality requirements.

\section{Event Selection}

Events are required to contain one isolated lepton (electron or muon) with transverse momentum $p_{\mathrm{T}}^{1}>25 \mathrm{GeV},|\eta|<2.5$ for muons, $|\eta|<2.47$ for electrons (excluding the calorimeter transition region $1.37<|\eta|<1.52$ ), missing transverse energy $E_{\mathrm{T}}^{\text {miss }}>25 \mathrm{GeV}$, and two jets with

\footnotetext{
${ }^{1}$ In the ATLAS coordinate system the pseudorapidity, $\eta$, is defined as $\eta=-\ln [\tan (\theta / 2)]$, where $\theta$ is measured with respect to the $z$-axis, defined to be parallel to the beam. The azimuthal angle, $\phi$, is measured with respect to the $x$-axis, which points towards the center of the LHC ring, and the y-axis points upwards.
} 
$p_{\mathrm{T}}>25 \mathrm{GeV}$ and $\left|\eta_{\text {jet }}\right|<2.5$, of which at least one is identified as a $b$-jet. For the SM s-channel analysis, jets containing $b$-quarks are tagged by reconstructing secondary vertices from the tracks associated with each jet and requiring a minimum signed decay length significance of the secondary vertices relative to the primary vertex (SV0). The chosen working point corresponds to a $b$-tagging efficiency of $50 \%$ and a light quark jet rejection factor of around 300, as measured in simulated topquark pair $(t \bar{t})$ events as shown in Fig. 2 . The $b$-tagging algorithm for the $t b$ resonances search uses measurements of the impact parameters of tracks and the properties of reconstructed vertices; these are combined in a neural network to extract a tagging decision for each jet (IP3D+JetFitter) [卂]. The working point is chosen at a $b$-tagging efficiency of $57 \%$, leading to a light quark jet rejection factor of around 600. Correction factors for the modeling of the $b$-tagging efficiency are derived from data [8]. Events before application of any $b$-tagging are referred to as pre-tagged events. Events where one or both jets are $b$-tagged are referred to as single- or double-tagged events, respectively.

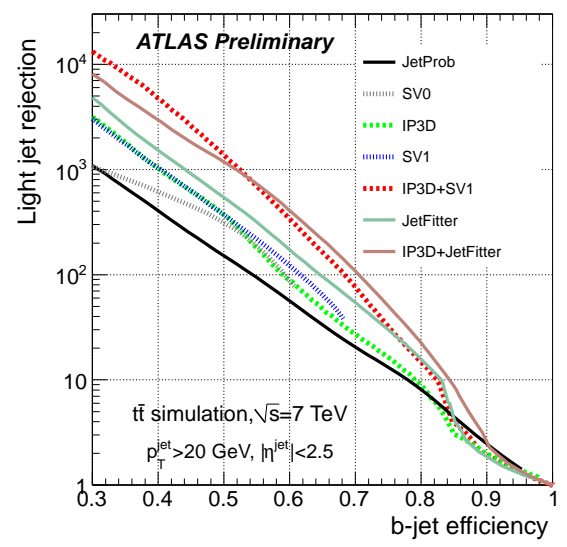

Figure 2: Light-jet rejection as a function of the $b$-jet tagging efficiency for the early tagging algorithms (JetProb and SV0) and for the high performance algorithms, based on simulated $t \bar{t}$ production events 仍.

\section{Background Estimation}

The background contributions consist of multijets, $W+$ jets and $t \bar{t}$ events, with smaller contributions from $Z+$ jets and diboson events. Data-driven methods and simulated samples are used to model backgrounds. The $t \bar{t}$, the $W t$ and $t$-channel single top, $Z+$ jets, $W W, W Z$ and $Z Z$ background contributions are normalized to their theory predictions, and their shape is taken from simulation. Since the multijets background is difficult to simulate precisely, its contribution is reduced with a dedicated cut, $m_{\mathrm{T}}(W)>60 \mathrm{GeV}-E_{\mathrm{T}}^{\text {miss }} . m_{\mathrm{T}}(W)$ is the lepton- $E_{\mathrm{T}}^{\text {miss }}$ transverse mass defined as $\sqrt{2 p_{\mathrm{T}}^{l} p_{\mathrm{T}}^{v}\left(1-\cos \left(\phi^{l}-\phi^{v}\right)\right)}$, with $l$ and $v$ the lepton and the neutrino, respectively, with the measured $\mathrm{E}_{\mathrm{T}}^{\mathrm{miss}}$ vector providing the neutrino information. The shape of the multijets background is taken from a data sample which requires a jet with a similar detector signature as an electron. This jet must have $p_{\mathrm{T}}>25 \mathrm{GeV}$, and 80-95\% of its energy deposited in the electromagnetic section of the calorimeter. The jet must also have at least four associated tracks, thus reducing the contribu- 
tion from converted photons. The multijets background normalization is estimated using a binned likelihood fit to the $E_{\mathrm{T}}^{\text {miss }}$ distribution performed after applying all selection cuts but the $E_{\mathrm{T}}^{\text {miss }}$ cut. A systematic uncertainty of $50 \%(100 \%)$ is assigned to the multijets normalization for the pre-tagged and single-tagged (double-tagged) samples. The distributions for the $W+$ jets background are taken from simulated samples, while their overall normalization and flavor composition are derived from data [9].

\section{5. s-channel Result}

The SM s-channel single top cross section is measured using a cut-based approach in a data set of integrated luminosity $0.70 \mathrm{fb}^{-1}$. After the preselection, a series of tighter requirements is applied in order to isolate the s-channel signal. The most powerful cut is the requirement of exactly two $b$-tagged jets, but cuts on other event kinematics are also applied. For example, on the transverse mass of the $W$ boson $\left(m_{\mathrm{T}}(W)<111 \mathrm{GeV} / \mathrm{c}^{2}\right)$ or on the reconstructed top-quark mass

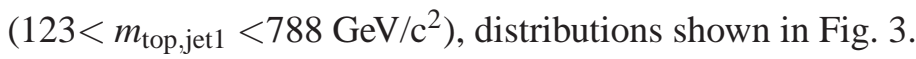
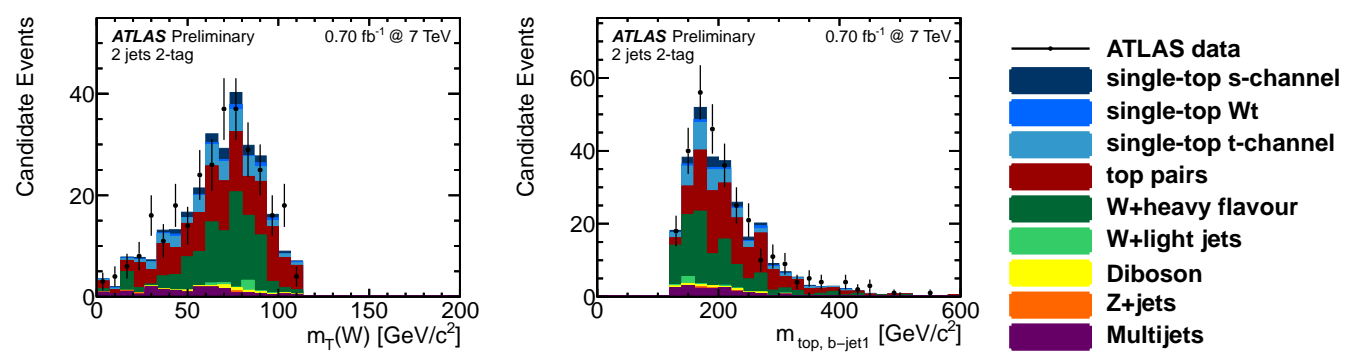

Figure 3: The transverse mass of the reconstructed $W$ boson (left) and the invariant mass of the leading $b$-tagged jet, the lepton and the neutrino (right) after the cut-based selection [9].

After the final selection, 296 data events are observed compared to a prediction of 285 . The predicted signal purity is $6 \%$. An upper limit on the SM s-channel production cross section of 26.5 $\mathrm{pb}$ is obtained at $95 \%$ confidence level, five times higher than the SM prediction. The main source of uncertainties are the data and MC statistics.

\section{6. tb Resonances Result}

These Proceedings also present a search for $t b$ resonances using an integrated luminosity of $1.04 \mathrm{fb}^{-1}$. A model with a right-handed $W_{R}^{\prime}$ boson with SM-like coupling strength [10] is chosen as the benchmark model for this search. The search exploits the shape of the invariant mass distribution $m_{t b}$ of the corresponding final state particles. Seven signal samples are simulated, with different $W_{R}^{\prime}$ boson mass assumptions, ranging from $500 \mathrm{GeV}$ to $2.0 \mathrm{TeV}$, as described in Table 1 . The respective signal cross section times branching ratio values are computed at next-to-leadingorder (NLO) [11], using CTEQ6.6 PDFs [12].

Figure 4 shows the $m_{t b}$ distribution for data and expected background for single- and doubletagged two-jet events together with the $W_{R}^{\prime}$ boson signal $m_{t b}$ distribution normalized to ten times the theoretical cross section. 


\begin{tabular}{rr}
\hline \hline$m_{W_{R}^{\prime}}[\mathrm{GeV}]$ & $\sigma \times B[\mathrm{pb}]$ \\
\hline 500 & $54.6 \pm 2.1$ \\
750 & $10.9 \pm 0.6$ \\
1000 & $2.92 \pm 0.18$ \\
1250 & $0.91 \pm 0.07$ \\
1500 & $0.31 \pm 0.03$ \\
1750 & $0.11 \pm 0.01$ \\
2000 & $0.044 \pm 0.005$ \\
\hline \hline
\end{tabular}

Table 1: $\quad W_{R}^{\prime}$ production cross section times branching ratio, $\sigma\left(p p \rightarrow W_{R}^{\prime}\right) \times B\left(W_{R}^{\prime} \rightarrow t b\right)$, at NLO in $p p$ collisions at $7 \mathrm{TeV}$ center-of-mass energy [1]]. The uncertainties include statistical, $\alpha_{s}$, renormalization and factorization scales, and PDF parametrization.
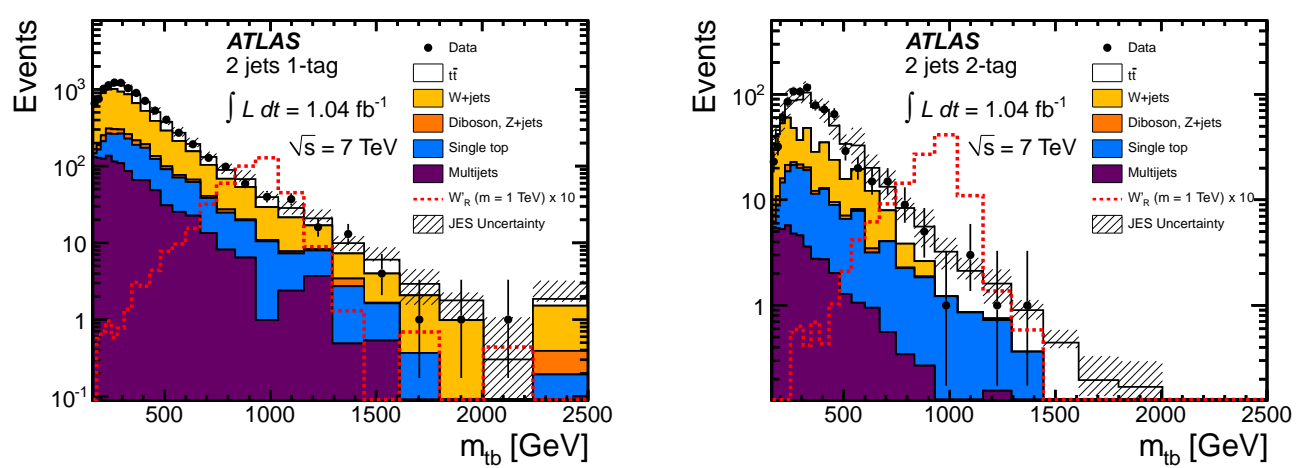

Figure 4: The distribution of $m_{t b}$ for single-tagged (left) and double-tagged (right) two-jet events in data compared to SM expectations. The expected $W_{R}^{\prime}$ boson signal has been normalized to ten times the theoretical cross section. The effect of the jet energy scale (JES) uncertainty on the predicted background is shown. The bin width is constant in $\log \left(m_{t b}\right)$. The last bin in each plot includes overflows [13].

Systematic uncertainties from various sources affecting background and signal acceptance, as well as shape changes in the invariant mass distribution are considered. The jet energy scale and the uncertainty on the $b$-tagging scale factors are the dominant systematic uncertainties for the signal. The background normalization yields are the dominant systematic uncertainty for the background contributions.

No statistically significant excess of events is observed in data, and upper limits on the cross section times branching ratio of $W_{R}^{\prime}$ resonances at 95\% credibility-level (CL) lie in the range 6.1$1.0 \mathrm{pb}$ for $W_{R}^{\prime}$ boson masses ranging from 0.5 to $2.0 \mathrm{TeV}$ [13]. These limits are translated into a lower bound on a right-handed $W_{R}^{\prime}$ boson mass, giving $m_{W_{R}^{\prime}}>1.13 \mathrm{TeV}$ at $95 \% \mathrm{CL}$. Figure 5 shows observed and expected limits from single- and double-tagged events combined.

\section{Conclusions}

The latest ATLAS results on searches in the $t b$ final state have been presented. Both analyses are based on a common event selection and background estimation techniques. Work is already in 


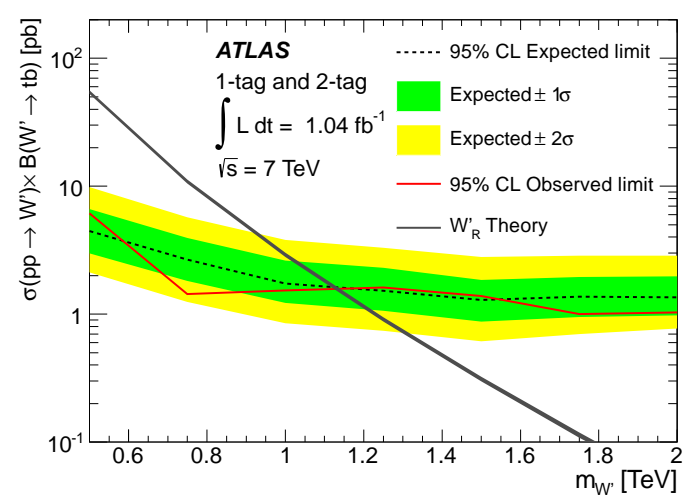

Figure 5: 95\% CL limit on the cross section, $\sigma\left(p p \rightarrow W_{R}^{\prime}\right)$, times branching ratio for $W_{R}^{\prime} \rightarrow t b$ as a function of the $W^{\prime}$ boson mass. The theory curve is also shown [13].

progress to update these results including new approaches and all the data collected by the ATLAS detector during the 2011 and 2012 LHC runs.

\section{References}

[1] Tim M.P. Tait, C.-P. Yuan, Phys. Rev. D 63, 014018 (2000).

[2] ATLAS Collaboration, J. Inst. 3, S08003 (2008).

[3] ATLAS Collaboration, Eur. Phys. J. C 72, 1849 (2012).

[4] N. Kidonakis, Phys. Rev. D 83, 091503 (2011).

[5] N. Kidonakis, Phys. Rev. D 82, 054018 (2010).

[6] N. Kidonakis, Phys. Rev. D 81, 054028 (2010).

[7] ATLAS Collaboration, ATLAS-CONF-2011-102, https://cdsweb.cern.ch/record/1369219

[8] ATLAS Collaboration, ATLAS-CONF-2011-089, https://cdsweb.cern.ch/record/1356198

[9] ATLAS Collaboration, ATLAS-CONF-2011-118, https://cdsweb.cern.ch/record/1376410

[10] G. Altarelli, B. Mele and M. Ruiz-Altaba, Z. Phys. C 45, 109 (1989).

[11] Z. Sullivan, Phys. Rev. D 66, 075011 (2002).

[12] P. M. Nadolski et al., Phys. Rev. D 78, 013004 (2008).

[13] ATLAS Collaboration, Phys. Rev. Lett. 109, 081801 (2012). 